\title{
Propiedades mecánicas de tres marcas de arcos ortodóncicos de níquel-titanio termoactivados. Estudio in vitro
}

Mechanicals properties from three thermo-activated titanium-nickel orthodontic archwire brands. In vitro Study

\begin{abstract}
Resumen
La selección óptima de los alambres ortodóncicos permite al profesional realizar el tratamiento de manera eficiente; por ello, se requiere el conocimiento de las propiedades mecánicas básicas, las cuales no son reveladas por sus fabricantes. El estudio tuvo como objetivo comparar las propiedades mecánicas (tensión en la zona de activación constante, elongación en la zona de activación constante, resistencia máxima y límite de ruptura) de tres marcas de alambres ortodóncicos de níquel-titanio termoactivados. Se usaron 10 arcos ortodóncicos de níquel-titanio termoactivados de $0,4 \mathrm{~mm}$ de diámetro de las marcas: NiTi GACR (Grupo Control), Nitinol Termoactivado 3M UnitekR, Sentalloy GACR, Nitinol Termoactivado TECNIDENTR. Se prepararon cuerpos de prueba de $80 \mathrm{~mm}$ y fueron sometidos a ensayos de tracción utilizando una máquina Zwick / Roell modelo Z050 8402 de $50 \mathrm{kN}$ de capacidad, a una velocidad de $1 \mathrm{~mm} / \mathrm{min}$ y a $37^{\circ} \mathrm{C}$. Los resultados muestran el mayor valor fue obtenido por Nitinol $3 \mathrm{M}$ Unitek $(374,43+16,72 \mathrm{MPa})$ para la tensión en la zona de activación constante, mientras que Nitinol Tecnident mostró el mayor valor $(14,51+0,91 \%)$ en la elongación en la zona de activación constante, el Nitinol Sentalloy GAC registró el mayor valor en la resistencia máxima $(1398,59$ + 45,36 MPa) y límite de ruptura $(174,29+6,47 \mathrm{~N})$. Se concluye que los alambres ortodóncicos de níquel-titanio termoactivados, presentan diferencias en sus propiedades mecánicas.
\end{abstract}

Palabras clave: Alambres para ortodoncia. Ensayo de materiales. Resistencia a la tracción. Estudio comparativo.

\section{Abstract}

The optimal selection of orthodontic archwires allows the professional to make the treatment in an efficient way; therefore, the knowledge of basic mechanical properties is required, which are not revealed by their manufacturers. The purpose of this study was to compare the mechanical properties (constant tension in the activation area, constant elongation in the activation area, maximum resistance and break point) of three thermo-activated titanium-nickel orthodontic archwires. $100.4 \mathrm{~mm}$-diameter thermo-activated titaniumnickel orthodontic archwires from the following brands were used: NiTi GACR (Control Group), Nitinol Termoactivado 3M UnitekR, Sentalloy GACR, Nitinol Termoactivado TECNIDENTR. $80 \mathrm{~mm}$ test bodies were prepared and tested for traction using a Zwick / Roell machine model Z050 8402 with a $50 \mathrm{kN}$ capacity, at a speed of $1 \mathrm{~mm} / \mathrm{min}$ and at $37^{\circ} \mathrm{C}$. The results show that the higher value was obtained by Nitinol $3 \mathrm{M}$ Unitek $(374,43+16,72 \mathrm{MPa})$ for the constant tension in the activation area, while the Nitinol Tecnident showed the higher value $(14,51+0,91 \%)$ in the constant elongation in the activation area. The Nitinol Sentalloy GAC recorded the higher value in the maximum resistance $(1398,59+45,36 \mathrm{MPa})$ and break point $(174,29+6,47 \mathrm{~N})$. It was concluded that the thermo-activated titanium-nickel orthodontic archwires present differences in their mechanical properties.

Keywords: Orthodontic archwires. Material assay. Traction resistance. Comparative study.

\section{Introducción}

El profesional debe conocer las propiedades físicas, térmicas y mecánicas del material para escoger el alambre ortodóncico más apropiado, porque de ello depende parte del éxito del tratamiento. Esto se torna más importante con la aparición de nuevos materiales, los cuales presentan diferentes propiedades mecánicas.

Los alambres ortodóncicos de níqueltitanio proporcionan fuerzas leves $y$ continuas fisiológicamente más aceptables, siendo óptimas en las fases de alineamiento y nivelación, donde las fuerzas deben ser bien distribuidas $y$ controladas para evitar daños a los tejidos circundantes. ${ }^{2}$

Los alambres de níquel-titanio termoactivados presentan propiedades de superelasticidad y memoria de forma; a altos rangos de temperatura, las estructuras cristalinas de los alambres son cúbicas de cuerpo centrado (fase austenítica); y a baja temperatura, la estructura es hexagonal cerrada (fase martensítica). ${ }^{3}$

Las propiedades mecánicas de los alambres ortodóncicos no son dadas a conocer por el fabricante, por lo que no es posible conocer hasta qué punto pueden ser activados preservando así sus propiedades y eficiencia en el tratamiento. ${ }^{4}$
Artículo Original

\section{Viviana Ramos Torres ${ }^{1}$ Luciano Soldevilla Galarza ${ }^{2}$ Manuel Mattos Vela ${ }^{3}$}

\section{C.D. Práctica Privada}

2 Profesor asociado del Departamento Académico Estomatología Pediátrica

3 Jefe de práctica del Departamento Académico Estomatología Preventiva y Social

Facultad de Odontología de la UNMSM. Lima, Perú

* Tesis para optar el título de Cirujano Dentista

Correspondencia:

C.D. Viviana Milagros Ramos Torres

Dirección: Jr. Andrez Rázuri 480, VMT

(Teléfono: 295-0522)

E-mail: vivianamilagros@yahoo.es
Existen varios tipos de ensayos de laboratorio que pueden ser empleados para determinar las propiedades mecánicas, entre ellos, el más apropiado es el de tracción, ya que permite determinar varias propiedades y presenta un menor número de variables; y a pesar que no reproducen integralmente la situación clínica, establecen valores adecuados para la comparación entre los alambres. ${ }^{5,6}$

Por tanto, el objetivo del estudio fue comparar las propiedades mecánicas como: tensión el la zona de activación constante, elongación en la zona de activación constante, resistencia máxima y límite de ruptura de tres marcas de 
alambres ortodóncicos de níquel-titanio termoactivados.

\section{Material y Método}

Para el estudio, se utilizaron 10 arcos de níquel-titanio termoactivados de 0,4 mm de diámetro de las marcas comerciales: NiTi GAC (grupo control), Nitinol Termoactivado 3M Unitek, R Nitinol Sentalloy GAC, R y Nitinol Termoactivado TECNIDENT.

Los ensayos se realizaron siguiendo las normas de la Asociación Estándar de Australia, ${ }^{4}$ se prepararon 10 cuerpos de prueba de cada marca de arcos ortodóncicos conforme recibidos de fábri$\mathrm{ca}$, fueron cortados a $80 \mathrm{~mm}$ (mitad del arco), luego enderezados manualmente y marcados con un plumón indeleble, $30 \mathrm{~mm}$ en la zona central.

Los ensayos mecánicos fueron realizados en el Laboratorio de Pruebas Mecánicas del Área de Maquinaria de Planta del Centro Tecnológico Superior TECSUP; se empleó una máquina de ensayo mecánico Zwick / Roell (Zwick $\mathrm{GmbH} \&$ Co. KG in Ulm-Einsingen, Germany) modelo Z050 8402 de 50 $\mathrm{KN}$ de capacidad. La prueba fue supervisada por el Ingeniero Mecánico encargado de dicha área.

Los ensayos de tracción fueron realizados a $37^{\circ} \mathrm{C}$, el cual fue proporcionado por una cámara reguladora de temperatura que contenía un foco General Electric de 100 watts y un sensor próximo a la zona libre del alambre sujeto a la máquina.

Los 40 alambres cortados fueron fijados en las mordazas superior e inferior de la máquina, asegurándose que quedara en las marcas ya establecidas a los alambres y en la parte central de ambas mordazas a un mismo nivel. Se dio inicio al ensayo a una velocidad de deformación constante de $1 \mathrm{~mm} / \mathrm{min}$, con lo cual los alambres fueron sometidos a tracción, incrementándose gradualmente la tensión hasta la ruptura del mismo.

A través del computador, se obtuvo en simultáneo al ensayo, el registro de la tensión y deformación de cada alambre mediante la gráfica tensión-deformación. Obteniéndose: tensión en la zona de activación constante, elongación en la zona de activación constante, resistencia máxima y límite de ruptura.

El procesamiento y análisis de los datos se realizó en un computador Pentium 4 con Windows XP, mediante el Software SPSS versión 17 donde se realizaron cálculos de Media Aritmética y Desviación Estándar. Se empleó la prueba de ANOVA, seguida de la prueba de Tukey para realizar comparaciones múltiples entre las variables de estudio. Todas las pruebas se trabajaron a un nivel de confianza de $95 \%$.

\section{Resultados}

La mayor tensión promedio necesaria para llegar a la zona de activación constante la obtuvo el grupo de la marca Nitinol $3 \mathrm{M}$ con una media + desviación estándar de 374,43 + 16,72 $\mathrm{MPa}$, mientras que la menor tensión fue obtenida por el grupo de la marca Nitinol Tecnident con 291,89+23,18 MPa (Fig. 1). En el Análisis de Comparaciones Múltiples de Tukey, existen diferencias altamente significativas $(\mathrm{p}<$ $0,001)$ entre los grupos de Nitinol y el grupo control, y muy significativa $(\mathrm{p}<$ 0,01 ) entre los grupos Nitinol GAC y 3M (Tabla 1).

La mayor deformación promedio en la zona de activación constante la obtuvo el grupo Nitinol Tecnident con 14,5 + $0,91 \%$, mientras que el menor valor

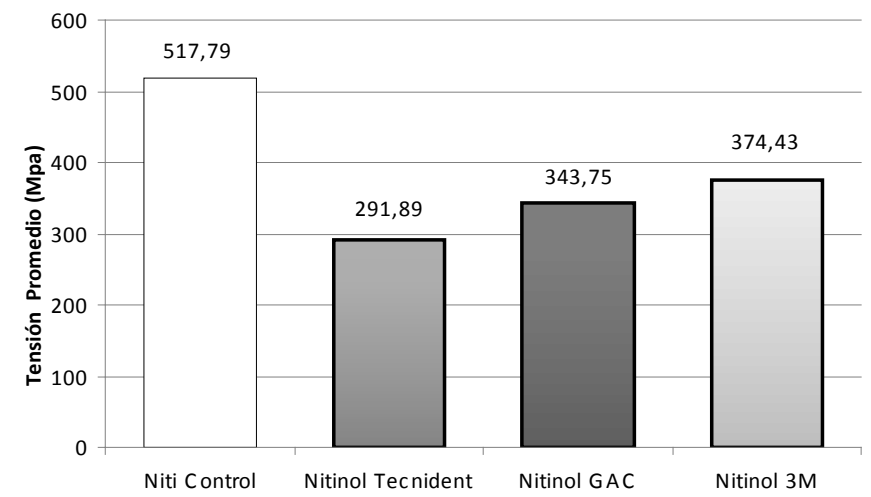

Fig 1. Tensión en la zona de activación constante de tres marcas de arcos ortodóncicos de níquel-titanio termoactivados y el grupo control a $37^{\circ} \mathrm{C}$.

Tabla 1. Comparaciones múltiples de la tensión en la zona de activación constante entre los grupos níquel- titanio termoactivados y control

\begin{tabular}{lccc}
\hline \multicolumn{1}{c}{ (I) GRUPO } & (J) GRUPO & $\begin{array}{c}\text { DIFERENCIA DE MEDIAS } \\
(\mathrm{I}-\mathrm{J})\end{array}$ & P \\
\hline Niti Control & Nitinol Tecnident & 225,90 & 0,000 \\
Niti Control & Nitinol GAC & 174,04 & 0,000 \\
Niti Control & Nitinol 3M & 143,35 & 0,000 \\
Nitinol Tecnident & Nitinol GAC & $-51,85$ & 0,000 \\
Nitinol Tecnident & Nitinol 3M & $-82,54$ & 0,000 \\
Nitinol GAC & Nitinol 3M & $-30,68$ & 0,008 \\
\hline
\end{tabular}

- Nivel de significancia: $p<0.05$ fue obtenido por el grupo Nitinol GAC con $11,17+0,91 \%$ (Fig. 2). Existen diferencias altamente significativas ( $\mathrm{p}$ $<0.001)$ entre los grupos de los arcos ortodóncicos de nitinol y el grupo control, y muy significativo ( $\mathrm{p}<0.01)$ entre el grupo de Nitinol Tecnident y $3 \mathrm{M}$ (Tabla 2).

La resistencia promedio máxima la obtuvo el grupo Nitinol GAC con $1398,59+45,36 \mathrm{MPa}$, mientras que el menor valor lo obtuvo el grupo Nitinol Tecnident con 1231,34 + 59,41 MPa. (Fig. 3) Existen diferencias altamente significativas $(\mathrm{p}<0.001)$ entre los grupos de arcos ortodóncicos, y muy significativa $(p<0.01)$ entre el grupo de Nitinol Tecnident y 3M (Tabla 3).

El mayor límite promedio de ruptura fue obtenida por el grupo Nitinol GAC con $174,29+6,47 \mathrm{~N}$, mientras que el menor valor fue obtenida por en el $9,98 \mathrm{~N}$ (Fig 4). Existen diferencias altamente significativas $(\mathrm{p}<0,001)$ entre los grupos de arcos ortodóncicos, y muy significativas $(p<0,01)$ entre los grupos de Nitinol Tecnident y 3M (Tabla 4). grupo Nitinol Tecnident con 150,06+ 


\section{Discusión}

Existen varios tipos de ensayos de laboratorio propuestos para determinar las propiedades mecánicas de los alambres ortodóncicos. "La American Dental Association (ADA) Specification N.er 32 for Orthodontic Wires Not Containing Precious Metals”, en 1977, estandarizó los ensayos de laboratorio para la clasificación de los alambres ortodóncicos, siendo seleccionados los ensayos de flexión y torsión, todavía los resultados obtenidos por los ensayos de tracción eran controversiales. Miura ${ }^{8}$ y Ashgarnia ${ }^{9}$ critican la precisión de los ensayos propuestos por la ADA obteniendo valores muy variables, principalmente para los de calibres menores a 0.020 ". Muench $^{10}$ indica que los ensayos de tracción son apropiados para los metales dúctiles usados en ortodoncia, permitiendo caracterizar perfectamente la superelasticidad, eligiendo por lo tanto los ensayos de tracción como los más confiables para la obtención de las propiedades mecánicas de los alambres, lo que justifica su elección para el presente trabajo de investigación.

En el presente estudio, se observó que la mayor tensión necesaria para llegar a la zona de activación constante fue para el grupo de Nitinol 3M Unitek (374,43 $+16,72 \mathrm{MPa}$ ) y Nitinol Sentalloy GAC $(343,75+15,37 \mathrm{MPa})$ mientras que la menor tensión fue obtenida por Nitinol Tecnident $(291,89+23,18 \mathrm{MPa})$, similares resultados a los obtenidos por Gravina (1) para el Nitinol GAC Sentalloy Medium de 0,018" con 377,78 $\mathrm{MPa}$ a $37^{\circ} \mathrm{C}$; lo cual confirma lo dicho por Uribe, ${ }^{11}$ quien menciona que a mayor diámetro mayor es la resistencia. Según Geoffrey, ${ }^{4}$ este punto es conocido como pseudo límite elástico, el cual es interpretado como la tensión requerida para el inicio de la fase martensitica (Ms), siendo clínicamente favorable, ya que permite bajas tensiones dentro de la fase Pseudoplástica, lo cual obtuvo para el Nitinol GAC Sentalloy Medium (0,016") $328 \mathrm{MPa}$ a $37^{\circ} \mathrm{C}$, debido a que la distancia entre las garras de la máquina de ensayos fue de 50 $\mathrm{mm}, 20 \mathrm{~mm}$ mayor al presente estudio, lo cual confirma lo dicho por Uribe, ${ }^{11}$ a mayor longitud de un alambre la resistencia disminuye. Según Geoffrey ${ }^{4}$ y $\mathrm{Gil}^{12}$ esta variable se interpreta clínicamente como la fuerza requerida para posicionar el alambre en el slot de los brackets siendo el 30 a $50 \%$ de dicha tensión manifestada para producir el movimiento ortodóncico de las piezas dentarias.

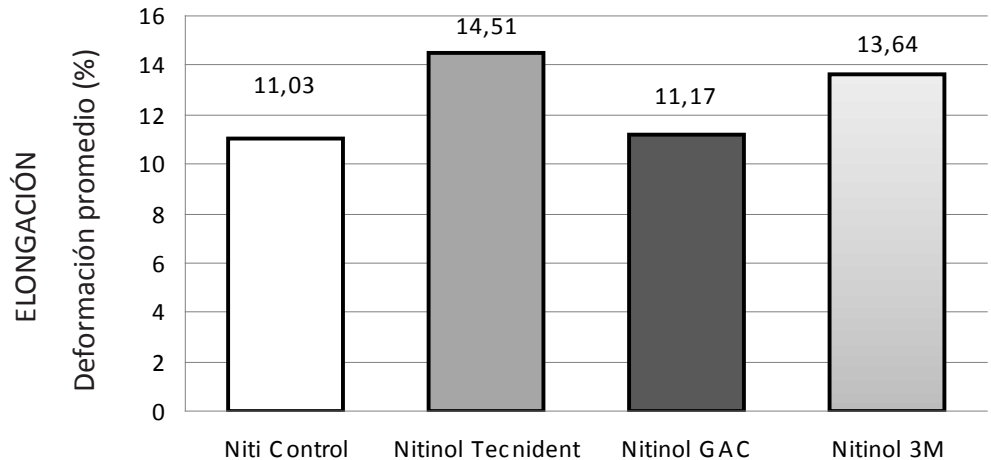

Fig 2. Elongación en la zona de activación constante de tres marcas de arcos ortodóncicos de níqueltitanio termoactivados y el grupo control a $37^{\circ} \mathrm{C}$.

Tabla 2. Comparaciones múltiples de la elongación en la zona de activación constante entre los grupos níquel- titanio termoactivados y control

\begin{tabular}{lccc}
\hline \multicolumn{1}{c}{ (I) GRUPO } & (J) GRUPO & $\begin{array}{c}\text { DIFERENCIA DE MEDIAS } \\
(\text { I-J) }\end{array}$ & P \\
\hline Niti Control & Nitinol Tecnident & $-3,48$ & 0,000 \\
Niti Control & Nitinol GAC & $-0,14$ & 0,952 \\
Niti Control & Nitinol 3M & $-2,61$ & 0,000 \\
Nitinol Tecnident & Nitinol GAC & 3,33 & 0,000 \\
Nitinol Tecnident & Nitinol 3M & 0,86 & 0,015 \\
Nitinol GAC & Nitinol 3M & $-2,47$ & 0,000 \\
\hline
\end{tabular}

- Nivel de significancia: $p<0,05$



Fig 3. Resistencia máxima de tres marcas de arcos ortodóncicos de níquel-titanio termoactivados y el grupo control a $37^{\circ} \mathrm{C}$.

Tabla 3. Comparaciones múltiples de la resistencia máxima entre los grupos níquel- titanio termoactivados y control

\begin{tabular}{lccc}
\hline \multicolumn{1}{c}{ (I) GRUPO } & (J) GRUPO & $\begin{array}{c}\text { DIFERENCIA DE MEDIAS } \\
\text { (I-J) }\end{array}$ & P \\
\hline Niti Control & Nitinol Tecnident & 270,28 & 0,000 \\
Niti Control & Nitinol GAC & 103,03 & 0,000 \\
Niti Control & Nitinol 3M & 227,41 & 0,000 \\
Nitinol Tecnident & Nitinol GAC & $-167,25$ & 0,000 \\
Nitinol Tecnident & Nitinol 3M & $-42,88$ & 0,118 \\
Nitinol GAC & Nitinol 3M & 124,38 & 0,000 \\
\hline - Nivel de significancia: $\mathrm{p}<0.05$ & & \\
\hline
\end{tabular}



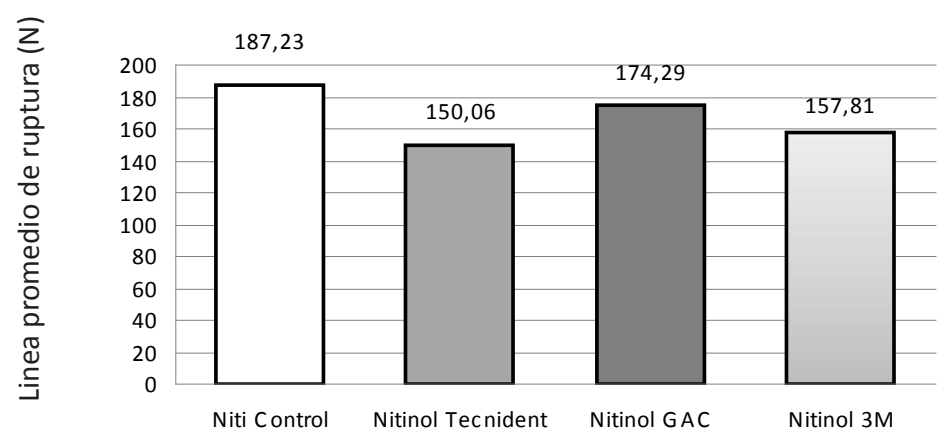

Fig 4. Límite de ruptura de tres marcas de arcos ortodóncicos de níquel-titanio termoactivados y el grupo control a $37^{\circ} \mathrm{C}$.

Tabla 4. Comparaciones múltiples del límite de ruptura entre los grupos níqueltitanio termoactivados y control.

\begin{tabular}{lccc}
\hline \multicolumn{1}{c}{ (I) GRUPO } & (J) GRUPO & $\begin{array}{c}\text { DIFERENCIA DE MEDIAS } \\
(\mathrm{I}-\mathrm{J})\end{array}$ & P \\
\hline Niti Control & Nitinol Tecnident & 37,16 & 0,000 \\
Niti Control & Nitinol GAC & 12,93 & 0,001 \\
Niti Control & Nitinol 3M & 29,41 & 0,000 \\
Nitinol Tecnident & Nitinol GAC & $-24,23$ & 0,000 \\
Nitinol Tecnident & Nitinol 3M & $-7,75$ & 0,064 \\
Nitinol GAC & Nitinol 3M & 16,47 & 0,000 \\
\hline
\end{tabular}

- Nivel de significancia: $p<0.05$

Se encontró que la mayor elongación en la zona de activación constante la obtuvo el grupo de Nitinol Tecnident y $3 \mathrm{M}$ Unitek $(14,51+0,91$ y $13,64+$ $0,38 \%$, respectivamente), mientras que el menor valor fue para Nitinol GAC Sentalloy $(11,17+0,50 \%)$, esta variable es interpretada según Geoffrey, ${ }^{4}$ Sakima ${ }^{13}$ y Gravina, ${ }^{1}$ como el rango de trabajo, es decir, almacenan cargas constantes por mayores intervalos de tiempo pudiendo ser deformados en mayor magnitud en comparación con aquellas que presenten menores porcentajes. Por lo tanto, las marcas Tecnident y 3M Unitek podrían presentar mayores rangos de trabajo y permanecer activos por más tiempo. Gravina1 encontró que los alambres de Nitinol de 0,018" 3M Unitek y GAC Sentalloy (12,52 y 9,68 \%, respectivamente) presentan una diferencia de $2,84 \%$, similar al presente estudio que fue de 2,47 \%; dicho autor concluye que el Nitinol 3M Unitek presentó el mayor porcentaje de elongación en la zona de activación constante, mientras que para el presente estudio, el Nitinol 3M Unitek ocupó el segundo lugar luego del Nitinol Tecnident.

El mayor resultado para la resistencia máxima fue obtenido por el grupo $\mathrm{Ni}$ tinol Sentalloy GAC $(1398,59+45,36$
$\mathrm{MPa}$ ), mientras que Nitinol 3M Unitek y Tecnident no presentaron diferencias estadísticamente significativas $(1274,21+29,32$ y $1231,34+59,41$ $\mathrm{MPa}$, respectivamente), resultado similar al obtenido por Gravina, ${ }^{1}$ el cual encontró para el Nitinol GAC Sentalloy (0,018") $1415,13 \mathrm{MPa}$ y para el Nitinol 3M Unitek (0,018”) 1011,44 MPa. Los alambres que presenten aún menor resistencia máxima son extremadamente altos cuando son comparadas clínicamente, lo cual coincide con lo mencionado por Burstone y cols., ${ }^{14}$ Miura y cols, ${ }^{8}$ y West y cols. ${ }^{2}$

El mayor límite de ruptura fue obtenido por el grupo Nitinol Sentalloy GAC, mientras que los menores valores no presentaron diferencias estadísticamente significativas en Nitinol Tecnident y 3M. Los alambres ortodóncicos usados en la clínica difícilmente presentarían el riesgo a la ruptura debido a que el profesional no emplea en la cavidad oral fuerzas de tal magnitud, confirmado por Gravina ${ }^{1}$, pero es necesario su conocimiento para determinar la tenacidad en un ensayo de tracción, también para obtener curvas de carga y descarga trabajadas bajo este límite.

Se observa que el grupo control (NiTi GAC) presentó los mayores valores con respecto a los arcos de níquel-titanio termoactivados, lo cual confirma lo mencionado por Gravina ${ }^{1}$, que los alambres de $\mathrm{Ni}$-Ti libres de $\mathrm{Cu}$ emplean mayores tensiones para alcanzar la zona constante de activación superiores a los alambres termoactivados $(\mathrm{Ni}$-Ti $\mathrm{Cu})$, también debido a que estos últimos presentan valores de Ms (inicio de la fase martensítica) más elevados, que coincide con lo dicho por Campista. ${ }^{15}$ Por el contrario, obtuvo el menor valor de elongación alcanzada en la zona de activación constante, lo cual confirma lo obtenido por Gravina ${ }^{1}$, quien obtuvo el menor porcentaje de deformación en la zona de activación constante para los alambres de NiTi GAC. También se confirma lo investigado por Sakima y cols., ${ }^{13}$ quienes observaron que los alambres termoactivados presentan mayores porcentajes de elongación en la zona de activación constante, en relación a los resultados obtenidos para los alambres superelásticos, lo cual es importante, pues los alambres termoactivados acumularían las mismas cargas por mayores intervalos de tiempo pudiendo ser deformados en mayor magnitud que los demás, acumulando fuerzas más uniformes durante las activaciones.

Todas las marcas de nitinol termoactivadas empleadas mostraron diferentes valores en sus propiedades mecánicas, debido al tratamiento térmico al cual han sido sometidos, así también, como la composición y proceso de manufactura, lo cual concuerda lo dicho por Kapila y col. ${ }^{16}$ y Yoneyama y cols. ${ }^{17}$

\section{Conclusiones}

1. La marca Nitinol $3 \mathrm{M}$ Unitek presentó la mayor tensión en la zona de activación constante, seguido por Nitinol Sentalloy GAC y Nitinol Tecnident, por lo cual la 3M Unitek podría emplearse en tratamientos con malposiciones dentarias que requieran altas fuerzas y la Nitinol Tecnident podría emplearse en tratamientos ortodóncicos con malposiciones dentarias que requieran ligeras fuerzas.

2. La marca Nitinol Tecnident obtuvo la mayor elongación en la zona de activación constante, seguido muy cerca por Nitinol 3M Unitek y Nitinol Sentalloy GAC.

3. La marca Nitinol Sentalloy GAC obtuvo la mayor resistencia máxima y límite de ruptura. 


\section{Referencias bibliográficas}

1. Gravina M. Fios ortodónticos: propriedades mecânicas deleventes e aplicaçao clínica. Rev. Dent. Press ortodon. Ortopedi. Facial. 2004; 9(1): 113-128.

2. West AE, Jones ML, Newcombe RG. Multiflex versus superelastic: A randomized clinical trial of the tooth alignment ability of initial arch wires. Am J of Orthod Dentofac Orthop. 1995; 108(5): 464-71.

3. Proffit WR. Ortodoncia. Teoría y Práctica, 2da. edición. Madrid - España: Editorial Mosby. 1994.

4. Geoffrey RS. A Comparison of the Tensile Properties of Nickel Titanium Orthodontic wires. Tesis para obtener el grado de Magíster. Australia: University Sydney; 1995.

5. Gurgel A, Kerr S, Powers M. Forcedeflection properties of superelastic nickel-titanium archwires. Am J of Orthod Dentofac Orthop. 2001; 120(4): 378-382.

6. New American Dental Association Specification N.er 32 for orthodontic wires not containing precious metals. Council on Dental Materials and Devices. J Am Dent Assoc. 1977; 95(6):1169-71.
7. Santoro M, Nicolay O, Cangialosi T. Pseudoelasticity and thermoelasticity of nikel-titanium alloy: a clinically oriented review. Part 1: Temperature transitional ranges. Am J of Orthod and Dentofac Orthop. 2001 Junio; 119(6):587-593.

8. Miura F. Magi, Ohura M. The Super-elastic property of japonese $\mathrm{NiTi}$ alloy use in orthodontics. Am $\mathrm{J}$ of Orthod and Dentofac Orthop. 1986; 90(1).

9. Asgharnia MK, Brantle Y, WA. Comparison of bending and tension tests for orthodontic wires. Am J of Orthod and Dentofac Orthop. 1986; 9(3): 28-36.

10. Muench A. Metais em Ortodoncia. Ortodontia bases para iniciação. 1994.

11. Uribe GA. Ortodoncia: Teoría y Clínica. Corporación para investigaciones Biológicas. 2004.

12. Gil FJ. Caracterización microestructural y mecánica de alambres de ortodoncia $\mathrm{Ni}-\mathrm{Ti}-\mathrm{Cu}$ con superelasticidad. Revista Iberoamericana de Ortodoncia. 1998 Enero-junio; 17(1): 9-18.

13. Sakima M, Dalstra M, Melsen B. How does temperature influence the properties of rectangular nickel-titanium wires? Eur J Orthod. 2006; 28(3): 282-291.

14. Burstone CJ, Qin B, Morton J Y. Chinese NiTi vire - A new orthodontic alloy. Am J of Orthod and Dentofac Orthop. 1985 Junio; 87(6): 445-452.

15. Campista CS. Comportamiento mecânico de ligas con efeito memoria. Tesis para obtener el grado de Magister. Ciencias en Ingienería, Metalúrgia y Materiales. RJ - Brazil: Universidad Federal de Rio de Janeiro; Marzo. 2005.

16. Kapila S, Sachdeva R. Mechanical properties and clinical applications of orthodontic wires. Am J of Orthod and Dentofac Orthop. 1989; 96(2): 100-09.

17. Yoneyama $\mathrm{T}$, Dol $\mathrm{H}$, Hamaeaka $\mathrm{H}$, Okamoto Y, Mogi M, Miura F. Super-elasticity and thermal behavior of $\mathrm{Ni}$-Ti alloy orthodontic archwires. Dent. Mater. J. 1992; 11(1):110.

Fecha de recepción: 11-5-10

Fecha de aprobación: 10-06-10 